\title{
Complexity of Indefinite Elliptic Problems
}

\author{
By Arthur G. Werschulz*
}

\begin{abstract}
This paper deals with the approximate solution of a linear regularly-elliptic $2 m$ th-order boundary-value problem $L u=f$, with $f \in H^{r}(\Omega)$ for $r \geq-m$. Suppose that the problem is indefinite, i.e., the variational form of the problem involves a weaklycoercive bilinear form. Of particular interest is the quality of the finite element method (FEM) of degree $k$ using $n$ inner products of $f$. The error of the approximation is measured in the Sobolev $l$-norm $(0 \leq l \leq m)$; we assume that $k \geq 2 m-1-l$. We assume that an a priori bound is known for either the Sobolev $r$-norm or for the Sobolev $r$-seminorm of $f$. We first consider the normed case. We find that the FEM has minimal error if and only if $k \geq 2 m-1+r$. Regardless of the values of $k$ and $r$, there exists a linear combination (called the spline algorithm) of the inner products used by the FEM which does have minimal error. For the seminormed case, we give a very restrictive condition which is necessary and sufficient for the error of the FEM to have a bound which is independent of $f$. When this condition holds, we find that the FEM has minimal error if and only if $k \geq 2 m-1+r$. However, we once again find that the spline algorithm (using the same inner products as does the FEM) has minimal error, no matter what values $k$ and $r$ have and regardless of whether the FEM has uniformly bounded error. We also show that the inner products used by the FEM is the best set of linear functionals to use.
\end{abstract}

1. Introduction. This paper is a theoretical study of the approximate solution of the elliptic boundary-value problem $L u=f$. We assume the problem is linear regularly-elliptic of $2 m$ th order with homogeneous boundary conditions, and that $f$ is defined on a region $\Omega \subset \mathrm{R}^{N}$. The smoothness of $f$ will be given by the assumption that $f \in H^{r}(\Omega)$, where $r \geq-m$. (See Section 2.) We want to approximate the solution $u$ by using the values of $n$ linear functionals of $f$, a typical example being the values of $f$ at $n$ points in $\Omega$. (See Section 3.)

We address two problems:

(i) Given the values of $n$ linear functionals of $f$, how may they be combined so as to approximate the solution of the elliptic problem with the smallest possible error?

(ii) What is the best set of $n$ linear functionals to use?

This paper is an extension of [15], where this problem was considered under the following conditions:

(i) The elliptic problem $L u=f$ is definite, i.e., its variational form involves a coercive bilinear form.

Received December 15, 1983; revised February 7, 1985.

1980 Mathematics Subject Classification. Primary $65 \mathrm{~N} 15,65 \mathrm{~N} 30,68 \mathrm{C} 05,68 \mathrm{C} 25$; Secondary $35 \mathrm{~J} 35,35 \mathrm{~J} 40$.

Key words and phrases. Indefinite elliptic problems, variational methods, finite element methods, optimal algorithms, computational complexity.

*This research was supported in part by the National Science Foundation under Grants MCS8203271 and MCS-8303111. 
(ii) The error of the approximate solution is measured by the energy norm, which is equivalent to the Sobolev $m$-norm.

(iii) An a priori bound is given on the Sobolev $r$-norm of $f$.

In [15], we studied the finite element method (FEM) of degree $k$, which uses $n$ inner products $\left(f, s_{1}\right), \ldots,\left(f, s_{n}\right)$, where $\left\{s_{1}, \ldots, s_{n}\right\}$ is a basis for a finite element subspace of degree $k$ and $(\cdot, \cdot)$ is the $L_{2}(\Omega)$-inner product. (See Section 4.) Here, we must assume $k \geq m$; otherwise, the FEM is not well-defined [15, Lemma 4.1]. The main result of [15] is that the FEM has minimal error (to within a constant) if and only if $k \geq 2 m-1+r$. We also prove that the set $\left\{\left(f, s_{1}\right), \ldots,\left(f, s_{n}\right)\right\}$ of inner products is always (to within a constant) the best choice of linear functionals. This holds for $\left\{s_{1}, \ldots, s_{n}\right\}$ a basis for a finite element subspace of degree $k$, for any value of $k \geq m$ and any value of $r$.

How crucial are the conditions in $[15]$ ?

Condition (i) disallows problems such as the Helmholtz problem: given $f \in$ $H^{r}(\Omega)$, find $u: \bar{\Omega} \rightarrow \mathrm{R}$ such that

$$
\begin{aligned}
\Delta u+\lambda u=f & \text { in } \Omega, \\
u=0 & \text { on } \partial \Omega,
\end{aligned}
$$

where $\lambda$ is not an eigenvalue of $-\Delta$. If $\lambda$ is bigger than the smallest eigenvalue of $-\Delta$, this problem does not yield a coercive bilinear form.

Condition (ii) is that the energy norm was used. Although this norm is equivalent to the Sobolev $m$-norm, the constant which measures this equivalence may be so large that a very good energy-norm solution may not be sufficiently accurate in the $m$-norm. (This would appear to be the situation in boundary-layer problems.) Moreover, it is sometimes of greater interest to use other norms, such as the $L_{2}(\Omega)$ norm for measuring displacement error.

Condition (iii) is needed to make the error of the approximate solution finite, and is implicitly used as a standard assumption for partial differential equations and finite element methods [1], [3], [8]. However, instead of assuming that the Sobolev $r$-norm of $f$ is bounded, one may only wish to assume an a priori bound on the Sobolev $r$-seminorm of $f$.

In this paper, the results of [15] are extended by weakening conditions (i), (ii), and (iii) above. We assume:

(i) ${ }^{\prime}$ The elliptic problem $L u=f$ is indefinite: its variational form involves a weakly coercive bilinear form.

(ii) ${ }^{\prime}$ Error is measured in the Sobolev $l$-norm, where $0 \leq l \leq m$.

(iii)' Both the normed and seminormed cases are considered, i.e., an a priori bound is given on the Sobolev $r$-norm or $r$-seminorm of $f \in H^{r}(\Omega)$ (respectively), the seminormed case making sense if and only if $r$ is a nonnegative integer.

Again, we will be interested in whether the FEM of degree $k$ has minimal error, and whether the inner products used by the FEM form the best set of linear functionals. We assume in this paper that

$$
k \geq 2 m-1-l
$$

see Section 3 for further discussion.

It turns out that in the normed case, replacing (i) by (i)' causes almost no difficulty, while replacing (ii) by (ii)' may be done via a variant of the Aubin-Nitsche duality argument [5, pp. 136-139]. Hence, in the normed case, the main results of [15] still hold; see Section 4 for details. 
The situation is different for the seminormed case. As an example, consider the problems

$$
-u^{\prime \prime}+u=f \quad \text { in }(0,1), \quad u(0)=u(1)=0,
$$

and

$$
-u^{\prime \prime}+u=f \quad \text { in }(0,1), \quad u^{\prime}(0)=u^{\prime}(1)=0
$$

where

$$
f \in H^{1}(0,1) \text { and } \int_{0}^{1}\left(f^{\prime}(x)\right)^{2} d x \leq 1 .
$$

For the problem (1.3), the error of the FEM is not uniformly bounded over all $f$ satisfying (1.5), no matter what the value of $k$ is. Despite this, there exists a linear combination of the inner products used by the FEM whose error is uniformly bounded over all such $f$. This linear combination is called the spline algorithm; see Section 3 for further discussion. In fact, the error of the spline algorithm goes to zero, independently of $f$, as the number of inner products goes to infinity. Moreover, there is no better choice of linear functionals for the problem (1.3) than the inner products which are used by the FEM.

For the problem (1.4), the situation is quite different. The FEM always has finite error and, when $k \geq 2$, its error is almost minimal. Once again, the inner products used by the FEM are (to within a constant factor) the best linear functionals, regardless of the value of $k \geq 1$.

We discuss the general seminormed case in detail in Section 5 . We show that the FEM has finite error if and only if $P_{r-1}(\Omega) \subseteq L S_{n}$, where $P_{r-1}(\Omega)$ is the restriction to $\Omega$ of the space of polynomials of degree at most $r-1$, and $S_{n}$ is the finite element subspace of dimension $n$. This condition is very restrictive; see Remark 6.1. When this condition holds, we find that the error of the FEM is (to within a constant factor) minimal if and only if $k \geq 2 m-1+r$. Regardless of whether the error of the FEM is minimal, there is a linear combination (called the spline algorithm) of the inner products used by the FEM whose error is minimal. Moreover, the inner products used by the FEM are (to within a constant factor) the best linear functionals, regardless of the value of $k \geq m$, and regardless of whether $P_{r-1}(\Omega) \subseteq L S_{n}$.

In Section 6, we discuss the complexity (i.e., the minimal cost) of obtaining approximate solutions whose error is less than $\varepsilon$. In the normed case, the FEM produces an approximation with minimal cost if and only if $k \geq 2 m-1+r$. If, however, $k<2 m-1+r$, the penalty for using the FEM (instead of the spline algorithm, which always produces an approximation with minimal cost) is unbounded as $\varepsilon \rightarrow 0$, for both the normed and seminormed cases. Since this is an asymptotic measure, we also wish to know whether there is a penalty for using the FEM for fixed $\varepsilon$ of moderate size when $k<2 m-1+r$. We consider a simple model problem, and show that the complexity of the spline algorithm is less than that of the FEM whenever $\varepsilon<\varepsilon_{0}$, where $\varepsilon_{0} \doteq 0.227$.

Finally, in Section 7, we summarize our work, and point out some possible extensions and open questions.

2. The Variational Boundary-Value Problem. In what follows, we use the standard notation for Sobolev spaces, inner products, and norms, for multi-indices, etc., found in [5]. Fractional- and negative-order Sobolev spaces are defined by 
Hilbert-space interpolation and duality, respectively; see [3, Chapter 2] and [8, Chapter 4] for details.

Let $\Omega \subset \mathbf{R}^{N}$ be a bounded, simply connected, $C^{\infty}$ region. Define the uniformly strongly elliptic operator

$$
L v=\sum_{|\alpha|,|\beta| \leq m}(-1)^{|\alpha|} D^{\alpha}\left(a_{\alpha \beta} D^{\beta} v\right),
$$

with real coefficients $a_{\alpha \beta} \in C^{\infty}(\bar{\Omega})$ such that $a_{\alpha \beta}=a_{\beta \alpha}$. In order to have appropriate boundary conditions, let

$$
B_{j} v=\sum_{|\alpha| \leq q_{j}} b_{j \alpha} D^{\alpha} v \quad(0 \leq j \leq m-1),
$$

where $b_{j \alpha} \in C^{\infty}(\partial \Omega)$ are real-valued and

$$
0 \leq q_{0} \leq \cdots \leq q_{m-1} \leq 2 m-1
$$

We assume that $\left\{B_{j}\right\}_{j=0}^{m-1}$ is a normal family of operators which covers $L$ on $\partial \Omega$. To make the boundary-value problem be selfadjoint, we let

and require that

$$
m^{*}=\min \left\{j: q_{j} \geq m\right\}
$$

$$
\left\{q_{j}\right\}_{j=0}^{m^{*}-1} \cup\left\{2 m-1-q_{j}\right\}_{j=m *}^{m-1}=\{0, \ldots, m-1\} .
$$

(See [3, Chapter 3] and [8, Chapter 5] for further definitions and illustrative examples.) We are interested in solving the elliptic boundary-value problem:

Given $f \in H^{r}(\Omega)$, where $r \geq-m$, find $u: \bar{\Omega} \rightarrow \mathbf{R}$ such that

$$
L u=f \quad \text { in } \Omega \text {, }
$$

$$
B_{j} u=0 \quad \text { on } \partial \Omega \quad(0 \leq j \leq m-1)
$$

Let

$$
H_{E}^{m}(\Omega)=\left\{v \in H^{m}(\Omega): B_{j} v=0\left(0 \leq j \leq m^{*}-1\right)\right\}
$$

denote the space of $H^{m}(\Omega)$-functions satisfying the essential boundary conditions. We define a symmetric, continuous bilinear form $B$ on $H_{E}^{m}(\Omega)$ by

$$
B(v, w)=\sum_{|\alpha|,|\beta| \leq m} \int_{\Omega} a_{\alpha \beta} D^{\alpha} v D^{\beta} w .
$$

In [15], we assumed that $B$ was $H_{E}^{m}(\Omega)$-coercive [8], i.e., that there exists a positive constant $\gamma$ such that

$$
B(v, v) \geq \gamma\|v\|_{m}^{2} \quad \forall v \in H_{E}^{m}(\Omega)
$$

When $m=0$, the conditions on $L$ yield that $B$ is $L_{2}(\Omega)$-coercive. However, for $m \geq 1$, there exist elliptic boundary-value problems which do not yield a bilinear form that is $H_{E}^{m}(\Omega)$-coercive (such as the Dirichlet problem for the Helmholtz equation).

In this paper, we assume instead that $B$ is weakly $H_{E}^{m}(\Omega)$-coercive [8, p. 310]. Since $B$ is symmetric, this means that there exists a positive constant $\gamma$ such that:

For any nonzero $v \in H_{E}^{m}(\Omega)$, there exists nonzero $w \in H_{E}^{m}(\Omega)$ such that

$$
|B(v, w)| \geq \gamma\|v\|_{m}\|w\|_{m} .
$$

The following lemma gives a condition which is sufficient to establish weak coercivity. The result appears to be well-known; its proof for arbitrary $m$ is a straightforward modification of the proof for the case $m=1$ which is found in [3, Chapter $5]$. 
LEMMA 2.1. Let $m \geq 1$. Suppose that

(i) the only solution of (2.1) with $f=0$ is $u=0$, and

(ii) $B$ is $\left[H_{E}^{m}(\Omega), L_{2}(\Omega)\right]$-coercive [8, p. 301]; that is, there exist $\gamma_{0}>0$ and $\gamma_{1} \geq 0$ such that

$$
|B(v, v)| \geq \gamma_{0}\|v\|_{m}^{2}-\gamma_{1}\|v\|_{0}^{2} \quad \forall v \in H_{E}^{m}(\Omega) .
$$

Then $B$ is weakly $H_{E}^{m}(\Omega)$-coercive.

Remark 2.1. Suppose that $B_{j}$ is the $j$ th normal derivative operator $(0 \leq j \leq$ $m-1)$, so that $(2.1)$ is a Dirichlet problem and $H_{E}^{m}(\Omega)=H_{0}^{m}(\Omega)$. Then $(2.3)$ is Gärding's inequality [1], which follows immediately from the conditions on $L$. Hence, $B$ is weakly $H_{0}^{m}(\Omega)$-coercive provided that (i) holds in Lemma 2.1. For example, the Helmholtz problem (1.1) is weakly $H_{0}^{1}(\Omega)$-coercive if $\lambda$ is not an eigenvalue of $-\Delta$.

We now define the variational boundary problem as follows. Let $r \geq-m$. We wish to solve the problem:

Given $f \in H^{r}(\Omega)$, find $u=S f \in H_{E}^{m}(\Omega)$ such that

$$
B(u, v)=(f, v)_{0}=\int_{\Omega} f v \quad \forall v \in H_{E}^{m}(\Omega) .
$$

From the Generalized Lax-Milgram Theorem [3, Theorem 5.2.1], $S$ is a Hilbert space isomorphism of $H^{-m}(\Omega)$ onto $H_{E}^{m}(\Omega)$, and so $S: H^{r}(\Omega) \rightarrow H_{E}^{m}(\Omega)$ is a bounded linear injection. Since $B$ is only assumed to be weakly coercive (i.e., we do not know that $B(v, v) \geq \gamma\|v\|_{m}^{2}$ holds), the problem (2.4) is said to be indefinite.

It is useful to recall the "shift theorem" ([3, Chapter 3], [8, Chapter 5]), which states that since $f \in H^{r}(\Omega)$, we have $S f \in H_{E}^{m}(\Omega) \cap H^{2 m+r}(\Omega)$, and that there exists a positive constant $\sigma$, which is independent of $f$, such that

$$
\sigma^{-1}\|S f\|_{2 m+r} \leq\|f\|_{r} \leq \sigma\|S f\|_{2 m+r} .
$$

If $r>N / 2$, then the shift theorem, Sobolev's embedding theorem, and an $m$-fold integration by parts yield that $u=S f$ is the solution to (2.1).

3. Finite Element Methods and Information. In this section, we discuss the finite element method (FEM). We describe the finite element information (FEI) which the FEM uses, and recall some quasi-optimality results from [15] for the case where assumptions (i), (ii), and (iii) from Section 1 hold.

We let $\left\{S_{n}\right\}_{n=1}^{\infty}$ be a regular family of finite element subspaces of degree $k$. That is, $S_{n}$ is an $n$-dimensional subspace of $H_{E}^{m}(\Omega)$ consisting of piecewise polynomials of degree $k$ over a triangulation $\tau_{n}$ of $\Omega$, where $\left\{\tau_{n}\right\}_{n=1}^{\infty}$ is regular [8, p. 132]. Of course, since $\Omega$ is $C^{\infty}$, we must make an additional assumption about boundary elements to guarantee that $S_{n} \subseteq H_{E}^{m}(\Omega)$ in the situation where $(2.1)$ is not a Neumann problem. (For instance, we may use curved elements as in [6].)

Remark 3.1. As indicated in (1.2) of the introduction, we assume that $k \geq$ $2 m-1-l$ in this paper. This technical assumption is needed for the proofs of some upper bounds which appear below. However, there are a number of situations where this holds automatically:

(i) If $\max \{0, m-1\} \leq l \leq m$, then (1.2) holds by [15, Lemma 4.1]. In particular, (1.2) holds when $m=0$ (where we have $l=0$ ) or when $m=1$ (in which case $l=0$ or $l=1)$.

(ii) If $N \geq 2$ and triangular elements are used, the results of [16] and the fact that $l \geq 0$ show that (1.2) holds. 
Hence, the only possibility that (1.2) will fail is when $N=1$ or when rectangular elements are used. However, it is possible for $k=m$ in either of these settings. Thus (1.2) can indeed fail to hold when $N=1$ or when rectangular elements are used.

The finite element method (FEM) using $\left\{S_{n}\right\}_{n=1}^{\infty}$ is defined as follows.

Let $f \in H^{r}(\Omega)$. Let $\left\{s_{1}, \ldots, s_{n}\right\}$ be a basis for $S_{n}$. Given $n$ inner products

$$
\mathcal{N}_{n} f=\left[\begin{array}{c}
\left(f, s_{1}\right)_{0} \\
\vdots \\
\left(f, s_{n}\right)_{0}
\end{array}\right] \text {, }
$$

choose $u_{n} \in S_{n}$ such that

$$
B\left(u_{n}, s_{i}\right)=\left(f, s_{i}\right)_{0} \quad(1 \leq i \leq n) .
$$

The FEM yields $u_{n}$ which depends only on $\mathcal{N}_{n} f$; we write $u_{n}=\varphi_{n}\left(\mathcal{N}_{n} f\right)$. We call $\mathcal{N}_{n}$ finite element information (FEI) of cardinality $n$.

We discuss some properties of the FEM. It is well-known (see, e.g., [3, Chapter 6] for the case $m=1$, the general case being similar) that if $\left\{\tau_{n}\right\}_{n=1}^{\infty}$ is quasi-uniform $\left[8\right.$, p. 272], then $B$ is weakly coercive on $\left\{S_{n}\right\}_{n=1}^{\infty}$ in the sense of [8, Theorem 8.1], and hence there exists a unique solution $u_{n} \in S_{n}$ to (3.1). Moreover, in this quasiuniform case, we may use (1.2) and [8, Theorems 8.2 and 8.6] to see that there exists a positive constant $C$ (independent of $f, u, n$, and $u_{n}$ ) such that

$$
\left\|S f-\varphi_{n}\left(\mathcal{N}_{n} f\right)\right\|_{m}=\left\|u-u_{n}\right\|_{m} \leq C \inf _{s \in S_{n}}\|u-s\|_{m}
$$

and

$$
\left\|S f-\varphi_{n}\left(\mathcal{N}_{n} f\right)\right\|_{l}=\left\|u-u_{n}\right\|_{l} \leq C n^{-(\mu+m-l) / N}\|f\|_{r} \quad \forall f \in H^{r}(\Omega),
$$

where

$$
\mu=\min \{k+1-m, m+r\} .
$$

We now relate the results of [15], where we assumed that (i), (ii), and (iii) from the Introduction held. These results, as well as those in the remainder of this paper, will be described using the $\Omega$ - and $\Theta$-notations, as well as the more standard $O$-notation: for functions $f$ and $g$, we write

$$
f=\Omega(g) \quad \text { iff } g=O(f)
$$

and

$$
f=\Theta(g) \text { iff } f=O(g) \text { and } g=O(f) .
$$

Define the error of the FEM as

$$
e_{m}\left(\varphi_{n}, \mathcal{F}\right)=\sup _{f \in \mathcal{F}}\left\|S f-\varphi_{n}\left(\mathcal{N}_{n} f\right)\right\|_{m}
$$

where $\mathcal{F}$ is the unit ball of $H^{r}(\Omega)$. Then

$$
e_{m}\left(\varphi_{n}, \mathcal{F}\right)=\Omega\left(n^{-\mu / N}\right) \quad \text { as } n \rightarrow \infty,
$$

where $\mu$ is as defined above. Furthermore, in the quasi-uniform case, the bound in (3.6) can be achieved, i.e.,

$$
e_{m}\left(\varphi_{n}, \mathcal{F}\right)=\Theta\left(n^{-\mu / N}\right) \quad \text { as } n \rightarrow \infty .
$$


We next ask whether there is any better way of combining the inner products in $\mathcal{N}_{n} f$, so as to minimize the error. Let

$$
e_{m}\left(\mathcal{N}_{n}, \mathcal{F}\right)=\inf _{\varphi} \sup _{f \in \mathcal{F}}\left\|S f-\varphi\left(\mathcal{N}_{n} f\right)\right\|_{m}
$$

Here $\varphi$ is any mapping (possibly nonlinear) which uses the inner products in $\mathcal{N}_{n} f$ to approximate $S f$. We say that $\varphi$ is an algorithm using $\mathcal{N}_{n}$. Thus $e_{m}\left(\mathcal{N}_{n}, \mathcal{F}\right)$ measures the minimal error among all algorithms using $\mathcal{N}_{n}$. We then have

$$
e_{m}\left(\mathcal{N}_{n}, \mathcal{F}\right)=\Omega\left(n^{-(m+r) / N}\right) \quad \text { as } n \rightarrow \infty .
$$

Furthermore, in the quasi-uniform case, the bound in (3.9) can be achieved, i.e.,

$$
e_{m}\left(\mathcal{N}_{n}, \mathcal{J}\right)=\Theta\left(n^{-(m+r) / N}\right) \quad \text { as } n \rightarrow \infty .
$$

From this, it follows that the FEM is quasi-optimal if and only if $k \geq 2 m-1+r$. By quasi-optimal [13], we mean that the FEM has error which (to within a constant factor) is minimal.

We finally ask whether there is any better set of inner products to use, so as to minimize the error. Let

$$
e_{m}(n, \mathcal{F})=\inf _{\mathcal{N}} e_{m}(\mathcal{N}, \mathcal{F}),
$$

the minimum being over all information $\mathcal{N}$ consisting of $n$ linear functionals. We then have

$$
e_{m}(n, \mathcal{F})=\Theta\left(n^{-(m+r) / N}\right) \quad \text { as } n \rightarrow \infty .
$$

From this it follows that the finite element information $\mathcal{N}_{n}$ is quasi-optimal.

4. Analysis of the Normed Case. In the next two sections, we extend the results of $[15]$ to the case where the bilinear form $B$ is weakly coercive and where error is measured in the norm $\|\cdot\|_{l}$ (where $0 \leq l \leq m$ and (1.2) holds). In this (as in the previous) section, we let $₹$ denote the unit ball in $H^{r}(\Omega)$. Moreover, we let $e_{l}\left(\varphi_{n}, \mathcal{F}\right), e_{l}\left(\mathcal{N}_{n}, \mathcal{F}\right)$, and $e_{l}(n, \mathcal{F})$ be as in the previous section, except that now " $m$ " is replaced by "l" in (3.5), (3.8), and (3.11).

We first determine the $n$th minimal error $e_{l}(n, \mathcal{F})$.

THEOREM 4.1.

$$
e_{l}(n, \mathcal{F})=\Theta\left(n^{-(r+2 m-l) / N}\right) \quad \text { as } n \rightarrow \infty .
$$

Proof. From [11], we have

$$
e_{l}(n, \mathcal{\Im})=d_{n}\left(S \mp, H_{E}^{l}(\Omega)\right) .
$$

(Here, and in what follows, we let $d_{n}$ denote the Kolmogorov $n$-width. In addition, a subscript $E$ attached to a Sobolev space indicates that those essential boundary conditions which are well-defined for that space are satisfied.) For any $\theta>0$, let

$$
X(\theta)=\left\{u \in H_{E}^{r+2 m}(\Omega):\|u\|_{r+2 m} \leq \theta\right\}=\theta B H_{E}^{r+2 m}(\Omega) .
$$

(Here, and in what follows, $B X$ denotes the unit ball of the space $X$.) Then the shift theorem (2.5) yields

$$
X\left(\sigma^{-1}\right) \subseteq S \mp \subseteq X(\sigma),
$$

and so (4.1) and (4.3) yield

$$
d_{n}\left(X\left(\sigma^{-1}\right), H_{E}^{l}(\Omega)\right) \leq e_{l}(n, \mathcal{F}) \leq d_{n}\left(X(\sigma), H_{E}^{l}(\Omega)\right) .
$$


But (4.2) implies that

$$
d_{n}(X(\theta))=\theta d_{n}\left(B H_{E}^{r+2 m}(\Omega), H_{E}^{l}(\Omega)\right) \quad \forall \theta>0,
$$

so that (4.4) and (4.5) yield

$$
\sigma^{-1} d_{n}\left(B H_{E}^{r+2 m}(\Omega), H_{E}^{l}(\Omega)\right) \leq e_{l}(n, \xi) \leq \sigma d_{n}\left(B H_{E}^{r+2 m}(\Omega), H_{E}^{l}(\Omega)\right) .
$$

From [3, Theorem 2.5.1], we find

$$
d_{n}\left(B H_{E}^{r+2 m}(\Omega), H_{E}^{l}(\Omega)\right)=\Theta\left(d_{n}\left(B H_{E}^{1 / 2}(\Omega), L_{2}(\Omega)\right)^{2(r+2 m-l)}\right) .
$$

But $H_{E}^{1 / 2}(\Omega)=H^{1 / 2}(\Omega)=H_{0}^{1 / 2}(\Omega)$, and so

$$
\begin{aligned}
d_{n}\left(B H_{E}^{1 / 2}(\Omega), L_{2}(\Omega)\right) & =d_{n}\left(B H_{0}^{1 / 2}(\Omega), L_{2}(\Omega)\right) \\
& =\Theta\left(d_{n}\left(B H_{0}^{1}(\Omega), L_{2}(\Omega)\right)^{1 / 2}\right),
\end{aligned}
$$

the last by another application of [3, Theorem 2.5.1]. Hence (4.6), (4.7), and (4.8) yield

$$
e_{l}(n, \Im)=\Theta\left(d_{n}\left(B H_{0}^{1}(\Omega), L_{2}(\Omega)\right)^{r+2 m-l}\right) .
$$

But the results of $[7]$ yield that

$$
d_{n}\left(B H_{0}^{1}(\Omega), L_{2}(\Omega)\right)=\Theta\left(n^{-1 / N}\right)
$$

The theorem follows immediately from (4.9) and (4.10).

We want to determine $e_{l}\left(\varphi_{n}, \mathcal{\exists}\right)$, the $H^{l}(\Omega)$-error of the FEM. Recall from (3.4) that $\mu=\min \{k+1-m, m+r\}$.

THEOREM 4.2. (i) $e_{l}\left(\varphi_{n}, \mathcal{F}\right)=\Omega\left(n^{-(\mu+m-l) / N}\right)$ as $n \rightarrow \infty$.

(ii) If $\left\{\tau_{n}\right\}_{n=1}^{\infty}$ is quasi-uniform, then

$$
e_{l}\left(\varphi_{n}, \mathcal{F}\right)=\Theta\left(n^{-(\mu+m-l) / N}\right) \quad \text { as } n \rightarrow \infty \text {. }
$$

Proof. First note that Theorem 4.1 yields

$$
e_{l}\left(\varphi_{n}, \mathcal{\exists}\right) \geq e_{l}(n, \mathcal{\exists})=\Theta\left(n^{-(r+2 m-l) / N}\right) .
$$

It remains to show that

$$
e_{l}\left(\varphi_{n}, \mathcal{F}\right)=\Omega\left(n^{-(k+1-l) / N}\right),
$$

since (4.11) and (4.12) yield (i), while (i) and the usual estimate (3.3) yield (ii).

The proof of $(4.12)$ is very similar to that of $[15,(4.17)]$. Let $\Omega^{0}$ be the interior of a hypercube whose closure is contained in $\Omega$, and let

$$
\tau_{n}^{0}=\left\{K \in \tau_{n}: K \in \overline{\Omega^{0}}\right\}
$$

Choose $u \in H_{E}^{r+2 m}(\Omega)$ such that

$$
u(x)=\frac{1}{(k+1) !} x_{1}^{k+1} \quad \forall x \in \overline{\Omega^{0}} .
$$

In what follows, we define (for any region $K \in \mathrm{R}^{n}$ ) $|\cdot|_{l, K}$ to be the usual seminorm $[5,(3.1 .2)]$ for nonnegative integers $l$, while for nonintegral values of $l \geq 0$, we define $|\cdot|_{l, K}$ by the Sloboditskiu technique $[8$, p. 96$]$, i.e.,

$$
|v|_{l, K}^{2}=\sum_{|\alpha|=\lfloor l\rfloor} \int_{K} \int_{K} \frac{\left(D^{\alpha} v(x)-D^{\alpha} v(\xi)\right)^{2}}{|x-\xi|^{N+2(l-\lfloor l\rfloor)}} d \xi d x
$$


In any case, we have $\|\cdot\|_{l, K} \geq|\cdot|_{l, K}$. We write $P_{k}(K)$ to denote the space of polynomials of degree up through $k$ over $K$.

Let $K \in \tau_{n}^{0}$. We claim that there is a positive constant $C_{1}$, independent of $K$ and $n$, such that

$$
\inf _{s \in P_{k}(K)}|u-s|_{l, K}^{2} \geq C_{1}^{2} \operatorname{vol}(K)^{2(k+1-l) / N+1} .
$$

To show (4.13) is true, note that $K$ is the affine image of a "reference element" $\hat{K}$ which is independent of $K$. It is straightforward to check that the functionals

$$
\hat{v} \mapsto|\hat{v}|_{k+1, \hat{K}} \quad \text { and } \quad \hat{v} \mapsto \inf _{\hat{s} \in P_{k}(\hat{K})}|\hat{v}-\hat{s}|_{l, \hat{K}}
$$

are seminorms on $P_{k+1}(\hat{K})$. Since $l \leq m \leq k$ (the last by [15, Lemma 4.1]), they have the same kernel $P_{k}(\hat{K})$. Since $P_{k+1}(\hat{K})$ is finite-dimensional, there is a positive constant $\hat{C}_{1}=\hat{C}_{1}(k, m, \hat{K})$ such that

$$
\inf _{\hat{s} \in P_{k}(\hat{K})}|\hat{v}-\hat{s}|_{l, \hat{K}} \geq \hat{C}_{1}|\hat{v}|_{k+1, \hat{K}} \quad \forall \hat{v} \in P_{k+1}(\hat{K}) .
$$

As in [15], we may then use [5, Theorem 3.1.2] and (4.14) to conclude that (4.13) holds.

Let

$$
\Omega_{n}=\operatorname{int} \bigcup\left\{K: K \in \tau_{n}^{0}\right\} .
$$

Letting $\# \tau_{n}^{0}$ denote the number of elements in the set $\tau_{n}^{0}$, we may then use (4.13) to see that

$$
\begin{aligned}
\inf _{s \in S_{n}}|u-s|_{l}^{2} & \geq \sum_{K \in \tau_{n}^{0}} \inf _{s \in P_{k}(K)}|u-s|_{l, K}^{2} \\
& \geq C_{1}^{2} \sum_{K \in \tau_{n}^{0}} \operatorname{vol}(K)^{2(k+1-l) / N+1} \\
& \geq C_{1}^{2} \frac{\operatorname{vol}\left(\overline{\Omega_{n}}\right)^{2(k+1-l) / N+1}}{\left(\# \tau_{n}^{0}\right)^{2(k+1-l) / N}}
\end{aligned}
$$

the last because

$$
\sum_{K \in \tau_{n}^{0}} \operatorname{vol}(K)=\operatorname{vol}\left(\overline{\Omega_{n}}\right) .
$$

Since $\Omega_{n} \subset \Omega^{0}$ and $\lim _{n \rightarrow \infty} \operatorname{vol}\left(\overline{\Omega_{n}}\right)=\operatorname{vol}\left(\overline{\Omega^{0}}\right)$, there is an index $n_{0}$ such that

$$
\operatorname{vol}\left(\overline{\Omega_{n}}\right) \geq \frac{1}{2} \operatorname{vol}\left(\overline{\Omega^{0}}\right) \quad \forall n \geq n_{0} .
$$

Hence (4.15) and (4.16) yield that there is a positive constant $C_{2}$, independent of $n$, such that

$$
\inf _{s \in S_{n}}|u-s|_{l} \geq C_{2}\left(\# \tau_{n}^{0}\right)^{-(k+1-l) / N} \quad \forall n \geq n_{0} .
$$

But $[15,(4.14)]$ shows that $\# \tau_{n}^{0}=O(n)$, and so there exists a positive constant $C_{3}$, independent of $n$, such that

$$
\inf _{s \in S_{n}}|u-s|_{l} \geq C_{3} n^{-(k+1-l) / N} \quad \forall n \geq n_{0} .
$$

Now let $f=L u$. Then $f$ is a nonzero element of $H^{r}(\Omega)$, since $L$ is injective and $u$ is a nonzero element of $H_{E}^{r+2 m}(\Omega)$. Let

$$
f^{*}=\frac{1}{\|f\|_{r}} f
$$


so that

$$
S f^{*}=\frac{1}{\|f\|_{r}} u
$$

Then $\varphi_{n}\left(\mathcal{N}_{n} f^{*}\right) \in S_{n}$ and $\|\cdot\|_{l} \geq|\cdot|_{l}$ yield

$$
\begin{aligned}
\left\|S f^{*}-\varphi_{n}\left(\mathcal{N}_{n} f\right)\right\|_{l} & \geq\left|S f^{*}-\varphi_{n}\left(\mathcal{N}_{n} f\right)\right|_{l} \geq \inf _{s \in S_{n}}\left|S f^{*}-s\right|_{l} \\
& \geq \frac{1}{\|f\|_{r}} \inf _{s \in S_{n}}|u-s|_{l}
\end{aligned}
$$

the latter since $S_{n}$ is a subspace of $H_{E}^{m}(\Omega)$. Letting

$$
C=\frac{C_{3}}{\|f\|_{r}}
$$

(which is a positive constant, independent of $n$ ), we see that (4.18) and (4.19) imply

$$
\left\|S f^{*}-\varphi_{n}\left(\mathcal{N}_{n} f\right)\right\|_{l} \geq C n^{-(k+1-l) / N} \quad \forall n \geq n_{0} .
$$

Since $f^{*} \in \mathcal{F}$, the desired conclusion (4.12) follows immediately from (4.20) and the definition (3.5) of the error of the FEM.

We now show that (in the quasi-uniform case) the FEM is a quasi-optimal method using FEI if and only if $k \geq 2 m-1+r$; however, FEI is always quasi-optimal information.

THEOREM 4.3. (i) $e_{l}\left(\mathcal{N}_{n}, \mathcal{F}\right)=\Omega\left(n^{-(r+2 m-l) / N}\right)$ as $n \rightarrow \infty$.

(ii) If $\left\{\tau_{n}\right\}_{n=1}^{\infty}$ is quasi-uniform, then

$$
e_{l}\left(\mathcal{N}_{n}, \mathcal{F}\right)=\Theta\left(n^{-(r+2 m-l) / N}\right) \text { as } n \rightarrow \infty \text {. }
$$

Proof. Using Theorem 4.1, we have

$$
e_{l}\left(\mathcal{N}_{n}, \mathcal{\Im}\right) \geq e_{l}(n, \mathcal{\exists})=\Theta\left(n^{-(r+2 m-l) / N}\right)
$$

establishing (i). To establish (ii), we let $z \in \mathcal{F} \cap \operatorname{ker} \mathcal{N}_{n}$, so that

$$
(z, s)_{0}=0 \quad \forall s \in S_{n} \text { and }\|z\|_{r} \leq 1 .
$$

Let $g \in H^{-l}(\Omega)$. Then $l \leq m$ yields that $g \in H^{-m}(\Omega)$. Symmetry of $B$ and (4.21) yield

$$
(S z, g)_{0}=B(S z, S g)=(z, S g)_{0}=(z, S g-s)_{0} \quad \forall s \in S_{n}
$$

and so

$$
\left|(S z, g)_{0}\right| \leq\|z\|_{r}\|S g-s\|_{-r} \quad \forall s \in S_{n}
$$

By $\left[3\right.$, Theorem 4.1.1], there exists a positive constant $C_{1}$, independent of $n$, as well as an $s \in S_{n}$, such that

$$
\|S g-s\|_{-r} \leq C_{1} n^{-\lambda / N}\|S g\|_{2 m-l} \leq C_{1} \sigma n^{-\lambda / N}\|g\|_{-l}
$$

Here, $\lambda$ is given by

$$
\lambda=\min \{r+2 m-l, k+1+r\}=r+2 m-l
$$

(the latter due to (1.2)), while $\sigma$ in the right-hand inequality comes from the shift theorem (2.5). Hence, (4.21)-(4.24) yield

$$
\frac{\left|(S z, g)_{0}\right|}{\|g\|_{-l}} \leq C_{1} \sigma n^{-(r+2 m-l) / N} .
$$


Since $g \in H^{-l}(\Omega)$ is arbitrary, (4.25) implies

$$
\|S z\|_{l}=\sup _{g \in H^{-l}(\Omega)} \frac{\left|(S z, g)_{0}\right|}{\|g\|_{-l}} \leq C_{1} \sigma n^{-(r+2 m-l) / N} .
$$

Since $z \in \mathcal{F} \cap \operatorname{ker} \mathcal{N}_{n}$ is arbitrary, (4.26) and [11, Chapters 2 and 3] yield that

$$
e_{l}\left(\mathcal{N}_{n}, \mathcal{F}\right)=\sup _{z \in \mathcal{F} \cap \operatorname{ker} \mathcal{N}_{n}}\|S z\|_{l} \leq C_{1} \sigma n^{-(r+2 m-l) / N} .
$$

The estimate (ii) follows immediately from (i) and (4.27).

Hence, in the quasi-uniform case, $\mathrm{FEI}$ is always quasi-optimal information, while the FEM is a quasi-optimal algorithm using FEI if and only if $k \geq 2 m-1+r$. It is known that for any information, there always exists a linear combination (of the linear functionals making up that information) whose error is minimal among all algorithms using that information; this algorithm is called the spline algorithm [11, Chapter 4]. In particular, this implies that the spline algorithm using FEI is always quasi-optimal among all algorithms using any information, while the FEM is quasioptimal among all algorithms using any information if and only if $k \geq 2 m-1+r$.

5. Analysis of the Seminormed Case. In the previous section, we extended the results of [15] to the case where the bilinear form $B$ is weakly coercive and error is measured in the norm $\|\cdot\|_{l}$ for $0 \leq l \leq m$. In this section, we weaken one further condition of [15]. Rather than assume that $\mathcal{F}=B H^{r}(\Omega)$, the unit ball of $H^{r}(\Omega)$, we assume in this section that $\mathcal{F}=B H^{r}(\Omega)$, the set of all functions $f$ in $H^{r}(\Omega)$ for which $|f|_{r} \leq 1$.

In this section, $e_{l}\left(\varphi_{n}, \mathcal{F}\right), e_{l}\left(\mathcal{N}_{n}, \mathcal{F}\right)$, and $e_{l}(n, \mathcal{F})$ will be defined as in the previous section, except that now $\mathcal{F}=B H^{r}(\Omega)$. We assume that $r$ is a nonnegative integer. Let $P_{r-1}(\Omega)$ denote the space of all polynomials in $N$ variables whose degree does not exceed $r-1$, treated as a space of functions defined on $\Omega$. We also let

$$
n^{*}=\operatorname{dim} P_{r-1}(\Omega)=\left(\begin{array}{c}
N+r-1 \\
r-1
\end{array}\right)
$$

denote the dimension of this space of polynomials.

We first obtain an estimate of the $n$th minimal error.

THEOREM 5.1. (i) If $n<n^{*}$, then $e_{l}(n, \mathcal{F})=\infty$.

(ii) $e_{l}(n, \mathcal{F})=\Theta\left(n^{-(r+2 m-l) / N}\right)$ as $n \rightarrow \infty$.

Proof. (i) follows immediately from [11, Theorem 2.3.2]. To establish (ii), let

$$
\hat{H}^{r}(\Omega)=H^{r}(\Omega) / P_{r-1}(\Omega)
$$

under the quotient norm. Then [14, Lemma 5.3] yields

$$
e_{l}(n, \mathcal{F})=O\left(e_{l}\left(n-n^{*}, B \hat{H}^{r}(\Omega)\right)\right)=O\left(e_{l}\left(n-n^{*}, B H^{r}(\Omega)\right)\right),
$$

where (as in the previous section) " $B$ " denotes the unit ball and the second step is because $B \hat{H}^{r}(\Omega) \subseteq B H^{r}(\Omega)$. But Theorem 4.1 yields

$$
e_{l}\left(n-n^{*}, B H^{r}(\Omega)\right)=\Theta\left(\left(n-n^{*}\right)^{-(r+2 m-l) / N}\right)=\Theta\left(n^{-(r+2 m-l) / N}\right) .
$$

Hence, (5.1) and (5.2) yield

$$
e_{l}(n, \mathcal{F})=O\left(n^{-(r+2 m-l) / N}\right) .
$$


On the other hand, $B H^{r}(\Omega) \subseteq B H^{r}(\Omega)=\mathcal{F}$ yields

$$
e_{l}(n, \mathcal{F}) \geq e_{l}\left(n, B H^{r}(\Omega)\right)=\Theta\left(n^{-(r+2 m-l) / N}\right) .
$$

The estimate (ii) then follows from (5.3) and (5.4).

We next investigate the error of the FEM. We show that either the FEM has infinite error, or the estimates of Theorem 4.2 hold. Once again, recall from (3.4) that $\mu=\min \{k+1-m, m+r\}$.

THEOREM 5.2. (i) If $S P_{r-1}(\Omega) \nsubseteq S_{n}$, then $e_{l}\left(\varphi_{n}, \mathcal{F}\right)=\infty$.

(ii) If $S P_{r-1}(\Omega) \subseteq S_{n}$ for all sufficiently large $n$, then

$$
e_{l}\left(\varphi_{n}, \mathcal{F}\right)=\Omega\left(n^{-(\mu+m-l) / N}\right) \quad \text { as } n \rightarrow \infty,
$$

where the " $\Omega$ " may be changed to " $\Theta$ " when $\left\{\tau_{n}\right\}_{n=1}^{\infty}$ is quasi-uniform.

Proof. (i) Let $S P_{r-1}(\Omega) \nsubseteq S_{n}$. Since the range of $\varphi_{n}$ is $S_{n}$, there exists $f \in$ $P_{r-1}(\Omega)$ such that $\varphi_{n}\left(\mathcal{N}_{n} f\right) \neq S f$. Since $\varphi_{n}$ is linear and $P_{r-1}(\Omega)=\operatorname{ker}|\cdot|_{r}$, we may use [11, Lemma 3.2.2] to see that $e_{l}\left(\varphi_{n}, \mathcal{F}\right)=\infty$.

(ii) Let $S P_{r-1}(\Omega) \subseteq S_{n}$. Since $B H^{r}(\Omega) \subseteq \mathcal{F}$, Theorem 4.2(i) yields

$$
e_{l}\left(\varphi_{n}, \mathcal{F}\right) \geq e_{l}\left(\varphi_{n}, B H^{r}(\Omega)\right)=\Theta\left(n^{-(\mu+m-l) / N}\right) .
$$

Suppose now, in addition, that $\left\{\tau_{n}\right\}_{n=1}^{\infty}$ is quasi-uniform. We need only show that

$$
e_{l}\left(\varphi_{n}, \mathcal{F}\right)=O\left(n^{-(\mu+m-l) / N}\right) \quad \text { as } n \rightarrow \infty .
$$

Let $f \in \mathcal{F}$. Then there exists a unique choice of

$$
f_{1} \in P_{r-1}(\Omega) \quad \text { and } \quad f_{2} \in \hat{H}^{r}(\Omega)=H^{r}(\Omega) / P_{r-1}(\Omega)
$$

such that

$$
f=f_{1}+f_{2} .
$$

Recall [5, Theorem 3.1.1] that there is a positive constant $C_{1}$ such that

$$
\|\cdot\|_{r} \leq C_{1}|\cdot|_{r} \text { on } \hat{H}^{r}(\Omega) .
$$

Since $f_{1} \in P_{r-1}(\Omega)$, and $f \in \mathcal{F}$, we have

$$
\left\|f_{2}\right\|_{r} \leq C_{1}\left|f_{2}\right|_{r}=C_{1}|f|_{r} \leq C_{1},
$$

so that (3.3) yields that there is a positive constant $C_{2}$ (independent of $n$ and $f$ ) such that

$$
\left\|S f_{2}-\varphi_{n}\left(N_{n} f_{2}\right)\right\|_{l} \leq C n^{-(\mu+m-l) / N}\left\|f_{2}\right\|_{r} \leq C_{2} n^{-(\mu+m-l) / N} .
$$

Now $f_{1} \in P_{r-1}(\Omega)$ and $S P_{r-1}(\Omega) \subseteq S_{n}$, so that $S f_{1} \in S_{n}$. Since $l \leq m$, we may use (3.2) to see that

$$
\left\|S f_{1}-\varphi_{n}\left(\mathcal{N}_{n} f_{1}\right)\right\|_{l} \leq\left\|S f_{1}-\varphi_{n}\left(\mathcal{N}_{n} f_{1}\right)\right\|_{m} \leq C \inf _{s \in S_{n}}\left\|S f_{1}-s\right\|_{m}=0 .
$$

Since $S, \varphi_{n}$, and $\mathcal{N}_{n}$ are linear, the estimates (5.6), (5.8), and (5.9) yield that

$$
\left\|S f-\varphi_{n}\left(\mathcal{N}_{n} f\right)\right\|_{l} \leq C_{2} n^{-(\mu+m-l) / N} .
$$

Since $f \in \mathcal{F}$ is arbitrary, this yields (5.5). 
Remark 5.1. We illustrate the different possibilities in Theorem 5.2 by considering the model problems (1.3) and (1.4), where we have $r=1$ and $m=1$. Hence we define two operators $S_{1}: H^{1}(0,1) \rightarrow H_{0}^{1}(0,1)$ and $S_{2}: H^{1}(0,1) \rightarrow H^{1}(0,1)$ by

$$
u=S_{1} f \text { satisfies } \begin{gathered}
-u^{\prime \prime}+u=f \text { in }(0,1) \\
u(0)=u(1)=0
\end{gathered} \quad \forall f \in H^{1}(0,1),
$$

and

$$
u=S_{2} f \text { satisfies } \begin{gathered}
-u^{\prime \prime}+u=f \quad \text { in }(0,1) \\
u^{\prime}(0)=u^{\prime}(1)=0
\end{gathered} \quad \forall f \in H^{1}(0,1) .
$$

(Note that $S_{1}$ and $S_{2}$ differ only in their boundary conditions.) We claim that the FEM has infinite error for $S_{1}$, but has finite error for $S_{2}$. Again, keep in mind that these problems are being solved for all $f \in H^{1}(0,1)$ such that $|f|_{1} \leq 1$.

To see that the FEM has infinite error for $S_{1}$, note that $S_{1}\left(P_{0}(0,1)\right)$ is spanned by the solution of the problem

$$
-z^{\prime \prime}+z=1 \quad \text { in }(0,1), \quad z(0)=z(1)=0,
$$

the solution of which is

$$
z(x)=1-\left(\frac{e-1}{e^{2}-1}\right) e^{x}-\left(\frac{e^{2}-e}{e^{2}-1}\right) e^{-x} .
$$

Since $z$ is not a piecewise polynomial, we find that $S_{1}\left(P_{1}(0,1)\right) \nsubseteq S_{n}$, no matter how big the degree $k$ of the subspace $S_{n}$ or the dimension $n$ of $S_{n}$ are. Hence, the FEM has infinite error for the problem $S_{1}$.

We now consider the problem $S_{2}$. We find that $S_{2}\left(P_{0}(0,1)\right)=P_{0}(0,1)$, since the only solution to

$$
-z^{\prime \prime}+z=1 \quad \text { in }(0,1), \quad z^{\prime}(0)=z^{\prime}(1)=0
$$

is

$$
z(x) \equiv 1 \text {. }
$$

Since [15, Lemma 4.1] yields that $k \geq 1$, and there are no essential boundary conditions for this problem, we have $S_{2}\left(P_{0}(0,1)\right) \subseteq S_{n}$ for all $n \geq 1$ and any choice of $k$. This shows that the FEM has finite error for the problem $S_{2}$. In fact, the error of the FEM for this problem using $S_{n}$ (in the quasi-uniform case) is $\Theta\left(n^{-(\chi-l)}\right)$, where $\chi=\min \{k+1,3\}$. Comparing Theorems 5.1 and 5.2, we see that the FEM is quasi-optimal for this problem if and only if $k \geq 2$.

Remark 5.2. Since $S_{n} \subset H_{E}^{m}(\Omega)$, the condition $S P_{r-1}(\Omega) \subseteq S_{n}$ is equivalent to the condition $P_{r-1}(\Omega) \subseteq L S_{n}$. For situations in which the explicit form of the solution is unknown (i.e., most cases arising in practice), it will generally be easier to verify whether $P_{r-1}(\Omega) \subseteq L S_{n}$ than whether $S P_{r-1}(\Omega) \subseteq S_{n}$.

Remark 5.3. The condition $S P_{r-1}(\Omega) \subseteq S_{n}$ is very restrictive, since it is not generally the case that the solution $u$ of the problem $L u=f$ (with $f$ polynomial) is a piecewise polynomial satisfying the boundary conditions. (For example, we saw in Remark 5.1 that the solution $u=S_{1} f$ of (1.3) with $f \equiv 1$ involves exponential functions.) It would be extremely unlikely to have $S P_{r-1}(\Omega) \subseteq S_{n}$ in most situations, especially when the boundary of $\Omega$ is complicated or the coefficients $a_{\alpha \beta}$ of the $L$ are nonpolynomial. Hence, we see that the FEM has finite error for the seminormed case only under exceptional circumstances.

Hence, there are situations in which the FEM has infinite error, no matter how big $k$ and $n$ are. Is this a feature of the FEM itself, or is it a feature of the 
information which the FEM uses? In the remainder of this section, we show that the fault lies with the FEM rather than with the FEI. In fact, we will show that FEI is quasi-optimal information.

In order to do this, we first establish

LEMMA 5.1. There exists a positive integer $n_{0}$ and a positive constant $C$ such that for any $n \geq n_{0}$,

$$
\|z\|_{r} \leq C|z|_{r} \quad \forall z \in \operatorname{ker} \mathcal{N}_{n} .
$$

Proof. If $r=0$, this is immediate.

Suppose now that $r \geq 1$. If the conclusion is false, then there is a subsequence $\left\{z_{n_{i}} \in \operatorname{ker} \mathcal{N}_{n_{i}}\right\}_{i=1}^{\infty}$ such that

$$
\left\|z_{n_{i}}\right\|_{r}=1 \text { and } \lim _{i \rightarrow \infty}\left|z_{n_{i}}\right|_{r}=0 .
$$

Following the proof of [8, Theorem 3.1.1], the Rellich-Kondrašov compactness theorem yields that there exists $z \in P_{r-1}(\Omega)$ and a subsequence, which we again denote $\left\{z_{n_{i}} \in \operatorname{ker} \mathcal{N}_{n_{i}}\right\}_{i=1}^{\infty}$, such that

$$
\lim _{i \rightarrow \infty} z_{n_{i}}=z \text { in } H^{r}(\Omega) \text { and thus in } L_{2}(\Omega) .
$$

Hence, we see that

$$
\|z\|_{r}=1 .
$$

We claim that $z=0$, contradicting (5.12). Indeed, let $\varepsilon>0$. Using denseness of $C_{0}^{\infty}(\Omega)$ in $L_{2}(\Omega)$, there exists $w \in C_{0}^{\infty}(\Omega)$ such that

$$
\|z-w\|_{0}<\frac{1}{3} \varepsilon
$$

Since $C_{0}^{\infty}(\Omega) \subseteq H_{E}^{m}(\Omega) \cap H^{1}(\Omega)$, the standard results (found in, e.g., [8, Chapter 6]) yield that there is a positive constant $C_{1}$ (independent of $z$ and $w$ ) such that for any $j>0$, there exists $w_{j} \in S_{j}$ for which

$$
\left\|w-w_{j}\right\|_{0} \leq C_{1} j^{-1 / N}|w|_{1} .
$$

Hence, there is an index $i_{0}(\varepsilon)$ such that for any $i \geq i_{0}(\varepsilon)$, there exists $w_{n_{i}} \in S_{n_{i}}$ satisfying

$$
\left\|w-w_{n_{i}}\right\|_{0} \leq \frac{1}{3} \varepsilon .
$$

From (5.11) and $\|\cdot\|_{0} \leq\|\cdot\|_{r}$, there is an index $i_{1}(\varepsilon)$ such that for any $i \geq i_{1}(\varepsilon)$, there exists $z_{n_{i}} \in \operatorname{ker} \mathcal{N}_{n_{i}}$ for which

$$
\left\|z-z_{n_{i}}\right\|_{0} \leq \frac{1}{3} \varepsilon
$$

Let $i_{2}(\varepsilon)=\max \left\{i_{0}(\varepsilon), i_{1}(\varepsilon)\right\}$. Then (5.13)-(5.15) and the triangle inequality yield

$$
\left\|z_{n_{i}}-w_{n_{i}}\right\|_{0} \leq \varepsilon \quad \forall i \geq i_{2}(\varepsilon) .
$$

But $z_{n_{i}} \in \operatorname{ker} \mathcal{N}_{n_{i}}=H^{r}(\Omega) \cap\left(L_{2}(\Omega) / S_{n_{i}}\right)$ and $w_{n_{i}} \in S_{n_{i}}$. Hence $\left(z_{n_{i}}, w_{n_{i}}\right)_{0}=0$, which, when combined with (5.16), yields

$$
\left\|z_{n_{i}}\right\|_{0}^{2} \leq\left\|z_{n_{i}}\right\|_{0}^{2}-2\left(z_{n_{i}}, w_{n_{i}}\right)_{0}+\left\|w_{n_{i}}\right\|_{0}^{2}=\left\|z_{n_{i}}-w_{n_{i}}\right\|_{0}^{2} \leq \varepsilon^{2} .
$$

Thus for any $\varepsilon>0$, there is an index $i_{2}(\varepsilon)$ for which

$$
\left\|z_{n_{i}}\right\|_{0} \leq \varepsilon \quad \forall i \geq i_{2}(\varepsilon) .
$$

Hence

$$
\lim _{i \rightarrow \infty} z_{n_{i}}=0 \text { in } L_{2}(\Omega) .
$$

From (5.11) and (5.17), we have $z=0$, the desired contradiction.

We are now ready to show that the FEI is always quasi-optimal information for the seminormed case. 
THEOREM 5.3. (i) $e_{l}\left(\mathcal{N}_{n}, \mathcal{F}\right)=\Omega\left(n^{-(r+2 m-l) / N}\right)$ as $n \rightarrow \infty$.

(ii) If $\left\{\tau_{n}\right\}_{n=1}^{\infty}$ is quasi-uniform, then

$$
e_{l}\left(\mathcal{N}_{n}, \mathcal{F}\right)=\Theta\left(n^{-(r+2 m-l) / N}\right) \quad \text { as } n \rightarrow \infty .
$$

Proof. Using Theorem 5.1, we have

$$
e_{l}\left(\mathcal{N}_{n}, \mathcal{F}\right) \geq e_{l}(n, \mathcal{F})=\Theta\left(n^{-(r+2 m-l) / N}\right)
$$

establishing (i). To prove (ii), let $z \in \mathcal{F} \cap \operatorname{ker} \mathcal{N}_{n}$. For any $g \in H^{-l}(\Omega)$, we use (4.22) to see that

$$
\left|(S z, g)_{0}\right| \leq\|z\|_{r} \inf _{s \in S_{n}}\|S g-s\|_{-r} .
$$

By (4.23) and (4.24), there is a positive constant $C_{1}$ (independent of $n, g$, and $z$ ) such that

$$
\inf _{s \in S_{n}}\|S g-s\|_{-r} \leq C_{1} n^{-(r+2 m-l) / N}\|g\|_{-l} .
$$

Since $z \in \mathcal{F}$, Lemma 5.1 implies that there is a positive constant $C_{2}$ (independent of $n$ and $z$ ) such that

$$
\|z\|_{r} \leq C_{2}|z|_{r} \leq C_{2} .
$$

Hence, (5.18)-(5.20) yield the existence of a positive constant $C$ (independent of $n$, $z$, and $g$ ) such that

$$
\frac{\left|(S z, g)_{0}\right|}{\|g\|_{-l}} \leq C n^{-(r+2 m-l) / N} .
$$

Taking the supremum in (5.21) over all nonzero $g \in H^{-l}(\Omega)$, we find

$$
\|S z\|_{l} \leq C n^{-(r+2 m-l) / N} \text {. }
$$

Since $z \in \mathcal{F} \cap \operatorname{ker} \mathcal{N}_{n}$ is arbitrary, (5.22) and [11, Chapters 2 and 3] yield

$$
e_{l}\left(\mathcal{N}_{n}, \mathcal{F}\right) \leq C n^{-(r+2 m-l) / N},
$$

which, along with (i), establishes (ii).

Hence when the grid sequence is quasi-uniform, FEI is always quasi-optimal information for the seminormed case. This means that the spline algorithm $[11$, Chapter 4] using FEI is always quasi-optimal among all algorithms using any information. This is to be contrasted with the fact that in the seminormed case, the error of the FEM need not even be finite; however, when the FEM has finite error, it is quasi-optimal among all algorithms using any information if and only if $k \geq 2 m-1+r$.

6. Complexity Analysis. In this section, we discuss the complexity (minimal cost) of finding $\varepsilon$-approximations to the solution of the variational boundary-value problem, as well as the penalty for using the FEM when $k<2 m-1+r$.

Let $\varepsilon>0$. An algorithm $\varphi$ produces an $\varepsilon$-approximation to the problem $(S, \mathcal{F})$ in the $H^{l}(\Omega)$-norm if $e_{l}(\varphi, \mathcal{F}) \leq \varepsilon$. The complexity $\operatorname{comp}(\varphi)$ of an algorithm $\varphi$ is defined via the model of computation discussed in [11, Chapter 5]. Informally, we assume that any linear functional required by $\varphi$ can be evaluated with finite cost $c_{1}$, and that the cost of an arithmetic operation is unity.

Recall that $\varphi_{n}$ denotes the FEM of degree $k$ using the FEI $N_{n}$ based on the finite element subspace $S_{n}$. We assume that $\left\{\tau_{n}\right\}_{n=1}^{\infty}$ is quasi-uniform, where $\tau_{n}$ 
is the triangulation of $\Omega$ upon which $S_{n}$ is based. Since the FEI $\mathcal{N}_{n}$ contains $n$ inner products, we find that

$$
\operatorname{comp}\left(\varphi_{n}\right) \geq n c_{1}:
$$

Throughout the remainder of this paper, we assume that

$$
\operatorname{comp}\left(\varphi_{n}\right)=\Theta(n) \text { as } n \rightarrow \infty .
$$

Remark 6.1. The assumption (6.1) is reasonable in either of two cases. In the first case, we actually assume the existence of an algorithm which can solve the linear system generated by the FEM, whose number of operations is linear in $n$, the size of the linear system. This condition holds in a number of special casesfinding such linear-time algorithms is still an open problem for the general case.

Alternatively, one may wish to make an assumption of preconditioning. That is, we assume that any computation which is independent of the right-hand side $f$ is done in advance, and not counted when determining the number of operations required when approximating the solution $u$ to the problem $L u=f$. To make this more precise, recall that the FEM is a linear algorithm, i.e., it produces a linear approximation to the exact solution $u(x)$ having the form $\sum_{i=1}^{n}\left(f, s_{i}\right)_{0} g_{i}(x)$, where $g_{1}, \ldots, g_{n} \in S_{n}$. Since $g_{1}, \ldots g_{n}$ are independent of $f$, they may be determined in advance. (This precomputation may be especially efficient in the case where the problem $L u=f$ is to be solved for many different right-hand sides $f$.) Hence, computing the value of the FEM at any point in $\Omega$ requires at most $n$ multiplications and $n-1$ additions, once the $n$ inner products $\left(f, s_{1}\right), \ldots,\left(f, s_{n}\right)$ have been evaluated. Thus (6.1) holds.

Let

$$
\operatorname{FEM}_{l}(\varepsilon, \mathcal{F})=\inf \left\{\operatorname{comp}\left(\varphi_{n}\right): n \text { is an index such that } e_{l}\left(\varphi_{n}, \mathcal{F}\right) \leq \varepsilon\right\}
$$

denote the cost of solving the problem in the $H^{l}(\Omega)$-norm using the FEM. From the results of Sections 4 and 5, along with (6.1), we find

THEOREM 6.1. (i) $\operatorname{FEM}_{l}\left(\varepsilon, B H^{r}(\Omega)\right)=\Theta\left(\varepsilon^{-N /(\mu+m+l)}\right)$ as $\varepsilon \rightarrow 0$, where $\mu=$ $\min \{k+1-m, m+r\}$.

(ii) If there exists no integer $n \geq 0$ for which $S P_{r-1}(\Omega) \subseteq S_{n}$, then

$$
\operatorname{FEM}_{l}\left(\varepsilon, B H^{r}(\Omega)\right)=\infty \quad \forall \varepsilon \geq 0 .
$$

(iii) If there exists an integer $n_{0} \geq 0$ such that $S P_{r-1}(\Omega) \subseteq S_{n}$ for $n \geq n_{0}$, then

$$
\operatorname{FEM}_{l}\left(\varepsilon, B H^{r}(\Omega)\right)=\Theta\left(\varepsilon^{-N /(\mu+m+l)}\right) \quad \text { as } \varepsilon \rightarrow 0 .
$$

As mentioned previously in Sections 4 and 5, for any information, there exists a linear algorithm (the spline algorithm) having minimal error. Let $\varphi_{n}^{s}$ denote the spline algorithm using the FEI $\mathcal{N}_{n}$. If we agree once more to accept the idea of preconditioning as discussed in Remark 6.1, we find

$$
\operatorname{comp}\left(\varphi_{n}^{s}\right)=\Theta(n) \quad \text { as } n \rightarrow \infty .
$$

We now let

$$
\operatorname{SPLINE}_{l}(\varepsilon, \mathcal{F})=\inf \left\{\operatorname{comp}\left(\varphi_{n}^{s}\right): n \text { is an index such that } e_{l}\left(\varphi_{n}^{s}\right) \leq \varepsilon\right\}
$$

denote the cost of solving the problem using the spline algorithm. Using (6.2), the minimal error properties of the spline algorithm, and the results of Sections 4 and 5 , 
we find

THEOREM 6.2. In both the normed case $\mp=B H^{r}(\Omega)$ and the seminormed case $\mp=B H^{r}(\Omega)$, we have

$$
\operatorname{SPLINE}_{l}(\varepsilon, \mathcal{F})=\Theta\left(\varepsilon^{-N /(r+2 m-l)}\right) \quad \text { as } \varepsilon \rightarrow 0 .
$$

We now wish to determine the minimal cost of solving the problem. Let

$\operatorname{COMP}_{l}(\varepsilon, \mathcal{F})=\inf \left\{\operatorname{comp}(\varphi): \varphi\right.$ is an algorithm for which $\left.e_{l}(\varphi, \xi) \leq \varepsilon\right\}$

denote the problem complexity, i.e., the inherent cost of solving the problem with error not exceeding $\varepsilon$. Using the results of the two previous sections, we then find

THEOREM 6.3. In both the normed case $\mathcal{F}=B H^{r}(\Omega)$ and the seminormed case $₹=B H^{r}(\Omega)$, we have

$$
\operatorname{COMP}_{l}(\varepsilon, \mathcal{F})=\Theta\left(\varepsilon^{-N /(r+2 m-l)}\right) \quad \text { as } \varepsilon \rightarrow 0 .
$$

Hence, we may draw the following conclusions:

COROLLARY 6.1. (i) The spline algorithm using the FEI is always quasi-optimal, for both the normed and seminormed cases.

(ii) The FEM is quasi-optimal for the normed case if and only if $k \geq 2 m-1+r$. If $k<2 m-1+r$, then

$$
\frac{\mathrm{FEM}_{l}\left(\varepsilon, B H^{r}(\Omega)\right)}{\operatorname{COMP}_{l}\left(\varepsilon, B H^{r}(\Omega)\right)}=\Theta\left(\left(\frac{1}{\varepsilon}\right)^{\lambda N}\right) \quad \text { as } \varepsilon \rightarrow 0,
$$

where

$$
\lambda=\frac{1}{k+1-l}-\frac{1}{r+2 m-l}>0
$$

so that

$$
\lim _{\varepsilon \rightarrow 0} \frac{\mathrm{FEM}_{l}\left(\varepsilon, B H^{r}(\Omega)\right)}{\operatorname{COMP}_{l}\left(\varepsilon, B H^{r}(\Omega)\right)}=\infty .
$$

(iii) The FEM is quasi-optimal for the seminormed case if and only if $k \geq 2 m-$ $1+r$ and $S P_{r-1}(\Omega) \subseteq S_{n}$ for all sufficiently large $n$. If $k<2 m-1+r$ and $S P_{r-1}(\Omega) \subseteq S_{n}$ for all sufficiently large $n$, then

$$
\frac{\mathrm{FEM}_{l}\left(\varepsilon, B H^{r}(\Omega)\right)}{\mathrm{COMP}_{l}\left(\varepsilon, B H^{r}(\Omega)\right)}=\Theta\left(\left(\frac{1}{\varepsilon}\right)^{\lambda N}\right) \quad \text { as } \varepsilon \rightarrow 0,
$$

where $\lambda$ is given by (6.3). In the case that $S P_{r-1}(\Omega) \nsubseteq S_{n}$ for all $n \geq 0$, we have

$$
\operatorname{FEM}_{l}\left(\varepsilon, B H^{r}(\Omega)\right)=\infty \quad \forall \varepsilon \geq 0 \text {. }
$$

Hence when $k$ is too small for a given value of $r$, there is an infinite asymptotic penalty for using the FEM instead of the spline algorithm. Corollary 6.1 implies that there is an $\varepsilon_{0}>0$ such that

$$
\operatorname{SPLINE}_{l}(\varepsilon, \mp)<\operatorname{FEM}_{l}(\varepsilon, \mp) \text { for } 0<\varepsilon<\varepsilon_{0} \text {. }
$$

What is the value of $\varepsilon_{0}$ ? If $\varepsilon_{0}$ is unreasonably small, it may turn out that it is more reasonable to use the FEM for "practical" values of $\varepsilon$. We determine the value of $\varepsilon_{0}$ for a model problem in

Example 6.1. Let $N=1, \Omega=(0, \pi), m=1, r=1, H_{E}^{1}(\Omega)=H_{0}^{1}(0, \pi)$, and consider the bilinear form $B: H_{0}^{1}(0, \pi) \times H_{0}^{1}(0, \pi) \rightarrow \mathbf{R}$ defined by

$$
B(v, w)=\int_{0}^{\pi} v^{\prime} w^{\prime} \quad \forall v, w \in H_{0}^{1}(0, \pi)
$$


Hence for $f \in H^{1}(0, \pi), u=S f$ is the variational solution to the problem

$$
-u^{\prime \prime}=f \quad \text { in }(0, \pi), \quad u(0)=u(\pi)=0 .
$$

We choose $S_{n}$ to be the $n$-dimensional subspace of $H_{0}^{1}(0, \pi)$ consisting of piecewise linear polynomials with nodes at $x_{j}=j \pi /(n+1)$ for $0 \leq j \leq n+1$, so that $k=1$.

We wish to determine $\varepsilon_{0}$ such that (6.4) holds with $l=1$ and $₹=B H^{1}(0, \pi)$. This is similar to [15, Example 6.1]. The only difference is that in [15], we measured error by the energy norm (which is the $H^{1}$-seminorm $|\cdot|_{1}$ ), while here we use the $H^{1}$-norm $\|\cdot\|_{1}$.

Using the Poincaré inequality

$$
\|\cdot\|_{0} \leq|\cdot|_{1} \text { on } H_{0}^{1}(0, \pi)
$$

(see [9]), we have

$$
|\cdot|_{1} \leq\|\cdot\|_{1} \leq \sqrt{2}|\cdot|_{1} \text { on } H_{0}^{1}(0, \pi) .
$$

Hence $[15,(6.25)$ and (6.32)] and this inequality imply

$$
e_{1}\left(\varphi_{n}, \mathcal{F}\right) \geq \frac{\pi}{\sqrt{12}(n+1)} \doteq \frac{0.90689968}{n+1}
$$

and

$$
e_{1}\left(\varphi_{n}^{s}, \mathcal{F}\right) \leq \frac{\sqrt{2}}{(n+1)^{2}} \doteq \frac{1.4142136}{(n+1)^{2}},
$$

where (as before) $\varphi_{n}$ denotes the FEM and $\varphi_{n}^{s}$ denotes the spline algorithm using FEI.

To proceed further, we require sharper versions of (6.1) and (6.2). First, note that since any algorithm which actually makes use of all of the inner products of $\mathcal{N}_{n}$ must perform at least $n-1$ arithmetic operations, we find (in particular) that

$$
\operatorname{comp}\left(\varphi_{n}\right) \geq n c_{1}+n-1 \text {. }
$$

Next, we accept the idea of preconditioning. In this case, we find that linearity of the spline algorithm implies that it may be evaluated at any point in $\Omega$ using at most $n$ multiplications and $n-1$ additions, once the inner products of $\mathcal{N}_{n}$ have been evaluated. Hence

$$
\operatorname{comp}\left(\varphi_{n}^{s}\right) \leq n c_{1}+2 n-1 .
$$

Using (6.5) and (6.7), we find that

$$
\operatorname{FEM}_{1}(\varepsilon, \mathcal{F}) \geq\left(c_{1}+1\right)\left(\frac{\pi}{\sqrt{12}} \varepsilon^{-1}-1\right)-1,
$$

while (6.6) and (6.8) yield

$$
\operatorname{SPLINE}_{1}(\varepsilon, \mathcal{\mp}) \leq\left(c_{1}+2\right)\left(\sqrt[4]{2} \varepsilon^{-1 / 2}-1\right)-1
$$

From these two inequalities, it follows that

$$
\operatorname{SPLINE}_{1}(\varepsilon, \mathcal{F})<\operatorname{FEM}_{1}(\varepsilon, \mathcal{F}) \text { if } 0<\varepsilon<\varepsilon_{0},
$$

where $\varepsilon_{0}$ is the smallest positive solution $\varepsilon$ of

$$
\left(c_{1}+1\right)\left(\frac{\pi}{\sqrt{12}} \varepsilon^{-1}-1\right)=\left(c_{1}+2\right)\left(\sqrt[4]{2} \varepsilon^{-1 / 2}-1\right)
$$


Some algebra yields

$$
\varepsilon_{0}=\varepsilon_{0}\left(c_{1}\right)=\left(\sqrt[4]{2}\left(\frac{1}{2} c_{1}+1\right)-\sqrt{\sqrt{2}\left(\frac{1}{2} c_{1}+1\right)^{2}-\frac{\pi}{\sqrt{12}}\left(c_{1}+1\right)}\right)^{2}
$$

We now examine the value of $\varepsilon_{0}\left(c_{1}\right)$ under various assumptions on the cost $c_{1}$ of evaluating a linear functional, noting that $\varepsilon_{0}\left(c_{1}\right)$ increases with (nonnegative) $c_{1}$. Clearly, $c_{1} \geq 0$, so that

$$
\varepsilon_{0}\left(c_{0}\right) \geq \varepsilon_{0}(0)=\left(\sqrt[4]{2}-\left(\sqrt{2}-\frac{\pi}{\sqrt{12}}\right)^{1 / 2}\right)^{2} \doteq 0.22747884
$$

This tells us that (6.9) holds for all $\varepsilon$ less than (roughly) 0.227 . Next, we assume that $c_{1} \geq 1$, i.e., that evaluating a linear functional is at least as hard as an arithmetic operation. (It would be hard to imagine otherwise.) In this case,

$$
\varepsilon_{0}\left(c_{0}\right) \geq \varepsilon_{0}(1)=\left(\frac{3}{2} \sqrt[4]{2}-\left(\frac{9}{4} \sqrt{2}-\frac{\pi}{\sqrt{3}}\right)^{1 / 2}\right)^{2} \doteq 0.37714081
$$

Finally, it is reasonable to assume that $c_{1}$ is very large, i.e., that evaluating a linear functional is much harder than an arithmetic operation [11, p. 85]. One may check that

$$
\lim _{c_{1} \rightarrow \infty} \varepsilon_{0}\left(c_{1}\right)=\frac{1}{24} \pi^{2} \sqrt{2} \doteq 0.58157202,
$$

giving an estimate of $\varepsilon_{0}\left(c_{1}\right)$ for large values of $c_{1}$.

Based on this example, it seems reasonable to conjecture that (6.4) generally holds for "reasonable" values of $\varepsilon_{0}$. However, see the discussion at the end of $[15$, Section 6] for some comments regarding this conjecture.

7. Summary, Extensions, and Open Questions. We have shown that FEI of degree $k \geq m$ is always quasi-optimal information for indefinite linear elliptic problems $L u=f$ under the following conditions:

(i) Error is measured in the Sobolev $l$-norm, where $0 \leq l \leq m$.

(ii) Either $\|f\|_{r} \leq 1$ (where $r \geq-m$ ) or $|f|_{r} \leq 1$ (where $r$ is a nonnegative integer).

(iii) $k \geq 2 m-1-l$.

However, the FEM is not always quasi-optimal among all algorithms using FEI. In the normed case $\|f\|_{r} \leq 1$, the FEM is quasi-optimal if and only if $k \geq 2 m-1+r$. In the seminormed case $|f|_{r} \leq 1$, the FEM has finite error if and only if the finite element subspace contains $S P_{r-1}(\Omega)$; if this holds, then the FEM is quasi-optimal if and only if $k \geq 2 m-1+r$. In the case where $k<2 m-1+r$, the asymptotic penalty for using the FEM is infinite.

What happens when we try to weaken the assumptions above?

One natural weakening of (i) is to allow $l$ to satisfy the inequality $l \leq m$. The proofs of Theorems 5.2(i) and 6.2(ii) (the lower bound for the FEM) do not hold, since there seems to be no natural definition of the Sobolev $l$-seminorm for negative $l$. In the case where $\left\{\tau_{n}\right\}_{n=1}^{\infty}$ is uniform and $H_{E}^{m}(\Omega)=H^{m}(\Omega)$ (i.e., a Neumann problem), the results in [10] show that these results do hold for negative $l$ in this special situation. We conjecture that this is true in general, i.e., the lower bounds for the FEM given in these theorems hold for any $l \leq m$. However, the other results in this paper do hold for any $l$ such that $l \leq m$, provided (iii) still holds. 
A further natural weakening of (i) is to allow the error to be measured in nonHilbert norms. For example, one may wish to consider sup-norm estimates, since it is often more important to be able to get pointwise estimates on the error than mean-square error estimates.

Condition (ii) may be weakened in a number of ways. Rather than use the norm (or seminorm) over $H^{r}(\Omega)$, we may use $\|\cdot\|_{r, p}$ or $|\cdot|_{r, p}$, the norm or seminorm in $W^{r, p}(\Omega)$. Alternatively, we may decide to use the norm in more complicated spaces, such as the Besov space $B_{p}^{r, s}(\Omega)$ (see [4]). If we let $₹$ be the set of elements in one of these spaces whose norm (or seminorm) is bounded by unity, is FEI still quasi-optimal information? When is the FEM quasi-optimal?

What happens when (iii) no longer holds? In this case, the bounds that may be established using the techniques of this paper are no longer tight. Although (iii) holds for $l \geq 0$ in most cases of practical interest, it is important to find out what happens when (iii) no longer holds, as is the case when rectangular elements are used or when error is measured in negative norms.

We finally mention that all of the results of this paper hold for a worst-case setting, in which the error is determined by the maximum value (over all elements of $\xi$ ) of the difference between the exact solution and the approximate solution. This is by no means the only possible model. As an example, Trojan [12] considers an asymptotic setting, in which a fixed (but arbitrary) element $f \in \overline{\mathcal{F}}$ is chosen, a sequence of functionals of $f$ is evaluated, and these values are used to produce an approximation of the solution $u$ of the problem $L u=f$. Other possible settings include an average setting and an asymptotic-average setting. How close is the FEM to being optimal in such settings?

Acknowledgments. Many thanks are due to B. Białecki, M. Dryja, J. F. Traub, G. Wasilkowski, and H. Woźniakowski for their valuable comments.

Division of Science and Mathematics

Fordham University/College at Lincoln Center

113 West 60th Street

New York, New York 10023

Department of Computer Science

Columbia University

New York, New York 10027

1. S. AGmon, Lectures on Elliptic Boundary Value Problems, Van Nostrand, Princeton, NJ, 1965.

2. P. M. Anselone \& P. J. LauRent, "A general method for the construction of interpolating or smoothing spline functions," Numer. Math., v. 12, 1968, pp. 66-82.

3. I. BABUŠKA \& A. K. AZIZ, "Survey lectures on the mathematical foundations of the finite element method," The Mathematical Foundations of the Finite Element Method with Applications to Partial Differential Equations (A. K. Aziz, ed.), Academic Press, New York, 1972, pp. 3-359.

4. P. L. BUTZER \& H. Berens, Semi-groups of Operators and Approximation, SpringerVerlag, Berlin, 1967.

5. P. G. CiARLet, The Finite Element Method for Elliptic Problems, North-Holland, Amsterdam, 1978.

6. P. G. Ciarlet \& P. A. RAviart, "Interpolation theory over curved elements," Comput. Methods Appl. Mech. Engrg., v. 1, 1972, pp. 217-249.

7. J. W. Jerome, "Asymptotic estimates of the $L_{2} n$-width," J. Math. Anal. Appl., v. 22, 1968, pp. 449-464. 
8. J. T. ODEN \& J. N. REDDY, An Introduction to the Mathematical Theory of Finite Elements, Wiley-Interscience, New York, 1976.

9. M. Schultz, Spline Analysis, Prentice-Hall, Englewood Cliffs, NJ, 1973.

10. G. STRANG \& G. FIX, "A Fourier analysis of the finite element variational method," Constructive Aspects of Functional Analysis, Part II, C.I.M.E., Rome, 1973.

11. J. F. Traub \& H. Woźniakowski, A General Theory of Optimal Algorithms, Academic Press, New York, 1980.

12. J. M. TROJAN, "Asymptotic model for linear problems," (In preparation).

13. L. B. WAHLBIN, "Quasi-optimality of the $H_{0}^{1}$ projection into finite element spaces," Lectures on the Numerical Solution of Partial Differential Equations: Proceedings of the Special Year in Numerical Analysis (I. Babuška, T.-P. Liu, and J. Osborn, eds.), Dept. of Math., Univ. of Maryland, College Park, MD, Lecture Notes \#20, 1981.

14. A. G. Werschulz, "Does increased regularity lower complexity?," Math. Comp., v. 42, 1984, pp. 66-93.

15. A. G. WERSChUlz, "Finite element methods are not always optimal," (Submitted for publication).

16. A. ŻENIŠEK, "Hermite interpolation on simplexes in the finite element method," Proceedings EquaDiff 9, J. E. Purkyně University, Brno, 1972, pp. 271-277. 